\title{
Self-organized near-field etching of the sidewalls of glass corrugations
}

\author{
T. Yatsui - K. Hirata • Y. Tabata $\cdot$ Y. Miyake $\cdot$ Y. Akita • \\ M. Yoshimoto - W. Nomura - T. Kawazoe • M. Naruse • \\ M. Ohtsu
}

Received: 9 April 2011 / Published online: 15 May 2011

(C) The Author(s) 2011. This article is published with open access at Springerlink.com

\begin{abstract}
Using soda-lime glass with a nano-stripe pattern as a test specimen, we demonstrated self-organized nearfield etching with a continuum-wave laser $(\lambda=532 \mathrm{~nm})$ light source. Atomic force microscopy confirmed that nearfield etching decreases the flank roughness of the corrugations as well as the roughness of the flat surface.
\end{abstract}

\section{Introduction}

Reduction of surface roughness $\left(R_{a}\right)$ to less than $1 \AA$ is necessary to minimize light-scattering loss in various applications, such as high-quality, extreme-UV optical components, high-power lasers, and ultra-short pulse lasers [1]. Although chemical-mechanical polishing (CMP) has been

T. Yatsui $(\bowtie) \cdot$ W. Nomura $\cdot$ T. Kawazoe $\cdot$ M. Naruse $\cdot$ M. Ohtsu School of Engineering, The University of Tokyo, Bunkyo-ku, Tokyo 113-8656, Japan

e-mail: yatsui@ee.t.u-tokyo.ac.jp

T. Yatsui · W. Nomura $\cdot$ T. Kawazoe $\cdot$ M. Naruse $\cdot$ M. Ohtsu Nanophotonics Research Center, The University of Tokyo, 2-11-16 Yayoi, Bunkyo-ku, Tokyo 113-8656, Japan

K. Hirata $\cdot$ Y. Tabata

SIGMA KOKI Co., Ltd., 1-19-9 Midori, Sumida-ku, Tokyo 130-0021, Japan

Y. Miyake $\cdot$ Y. Akita $\cdot$ M. Yoshimoto

Department of Innovative and Engineered Materials, Tokyo Institute of Technology, 4259-J2-46 Nagatsuta, Midori-ku, Yokohama, Kanagawa 226-8503, Japan

M. Naruse

National Institute of Information and Communications

Technology, 4-2-1 Nukui-kita, Koganei, Tokyo 184-8795, Japan used for flattening the surfaces [2], its capacity for reducing the surface roughness $R_{a}$ is limited to about $2 \AA$, where the value of $R_{a}$ is an arithmetical average of the absolute values of the surface height deviations, measured from the best-square fitted plane. This limitation on the reduction of $R_{a}$ originates with the polishing pad roughness values being as large as $10 \mu \mathrm{m}$, and polishing particle diameters as large as $100 \mathrm{~nm}$ in the slurry. To overcome this limitation, we have developed a non-contact, self-organized method of near-field etching, utilizing a phonon-assisted photochemical reaction [3-7], which has been successfully applied to reduce the value of $R_{a}$ to as little as $1.36 \AA$ [3]. To selectively etch away protrusions on a silica surface, a continuum-wave (CW) laser $(\lambda=532 \mathrm{~nm})$ is used to dissociate the $\mathrm{Cl}_{2}$ gas. Since this photon energy is lower than the absorption band edge energy of $\mathrm{Cl}_{2}(\lambda=400 \mathrm{~nm})$ [8], the conventional $\mathrm{Cl}_{2}$ adiabatic photochemical reaction is avoided. However, on a nanometer scale rough surface, an optical near-field is also generated. This optical near-field then excites the multiplemode phonon state. As a result, a quasi-particle, known as a localized exciton-phonon polariton (EPP) [6], is created by the coupling between the optical near-field and multiple phonons. Since this quasi-particle has higher photon energy than that of the incident light photon, it is able to excite a higher molecular vibrational state [5, 6]. Hence, $\mathrm{Cl}_{2}$ is selectively photo-dissociated to generate radical $\mathrm{Cl}^{*}$ atoms (Fig. 1(a)), which etch away the surface roughness. The etching begins automatically when the surface is illuminated by the light, and it proceeds in a self-organized manner. It also ceases automatically when the surface becomes flat enough to stop generating EPP (Fig. 1(b)).

In addition to possessing this unique organizing property, near-field etching is a non-contact method (thus eliminating the need for polishing pads), with anticipated applications to various three-dimensional surfaces, including concave and 
Fig. 1 Schematic diagram of near-field etching of ( $\mathbf{a}$ and $\mathbf{b}$ ) flat and (c and d) nano-striped substrates

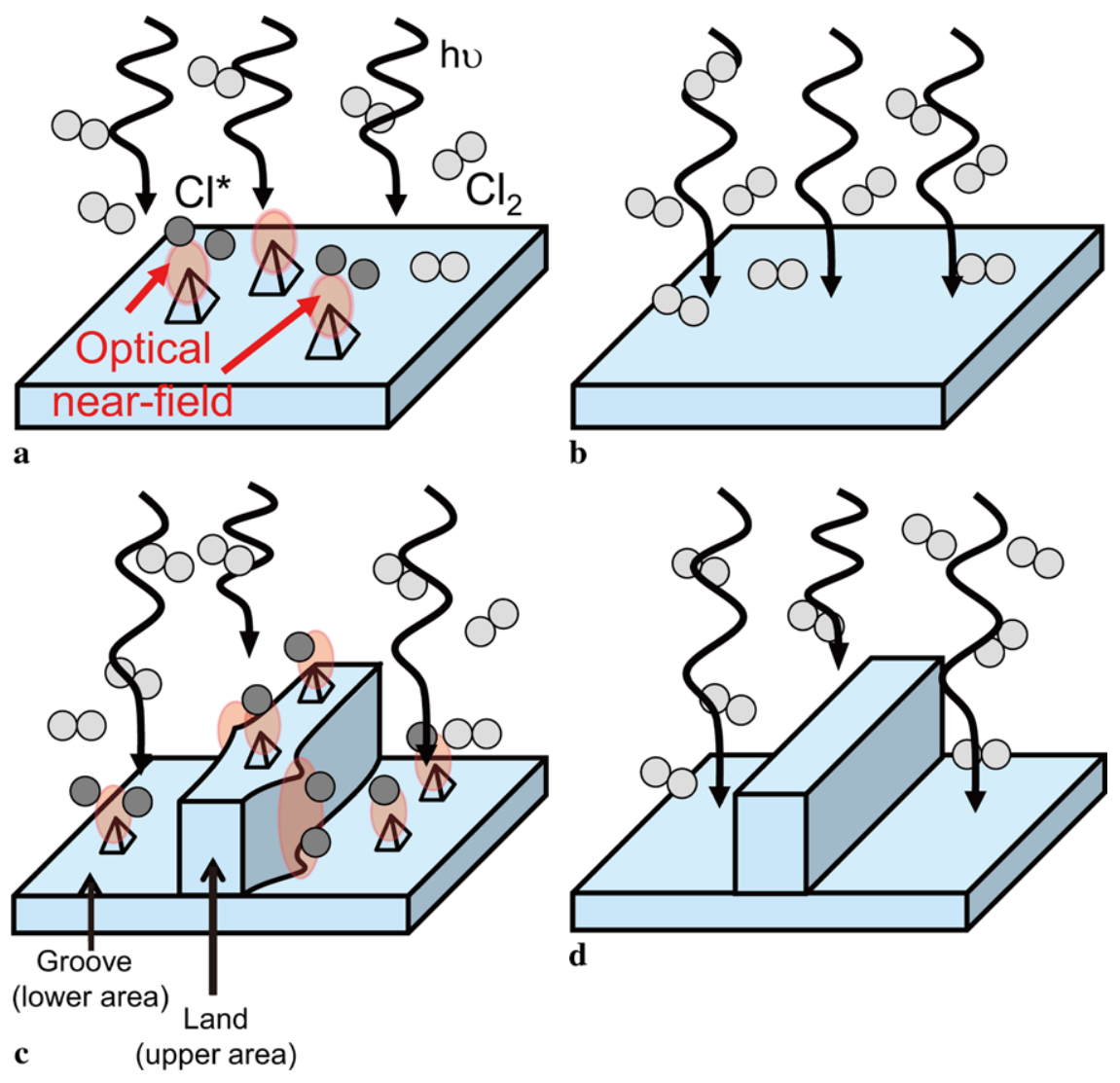

convex lenses, diffraction gratings, and the inner wall surfaces of a cylinder. To confirm this applicability, we utilized the procedure to smooth a substrate with nano-striped corrugation pattern (Figs. 1(c) and (d)).

\section{Experiment}

The nano-stripe pattern was fabricated on of a soda-lime glass specimen, using a thermal nanoimprint technique with $\mathrm{NiO}$ molds [9]. The $\mathrm{NiO}$ mold patterns were transferred to the soda-lime glass at $600^{\circ} \mathrm{C}$ for 5 minutes under 10 $\mathrm{MPa}$ of pressure. Figure 2(a) shows a typical atomic force microscopy (AFM) image of the soda-lime glass with the nano-stripe corrugation pattern. The average height and period of the stripes were $13.5 \mathrm{~nm}$ and $175 \mathrm{~nm}$, respectively.

Near-field etching was carried out by illuminating the substrate with a CW laser $(\lambda=532 \mathrm{~nm})$ having a spatially uniform power density of $0.28 \mathrm{~W} / \mathrm{cm}^{2}$. The $\mathrm{Cl}_{2}$ pressure in the chamber was maintained at $100 \mathrm{~Pa}$ at room temperature with a constant $\mathrm{Cl}_{2}$ flow rate of $100 \mathrm{sccm}$ (the same conditions used for smoothing a planner substrate of synthetic silica [3]). The surface morphology was evaluated via AFM after near-field etching. The scanning area of the AFM was $1.0 \mu m \times 1.0 \mu \mathrm{m}$, and the scanned area was
256 ( $x$-axis) $\times 256$ ( $y$-axis) pixels, with a spatial resolution of $4 \mathrm{~nm}$ for each axis.

\section{Results and discussion}

Figure 2(b) shows a typical AFM image after 30 minutes of near-field etching. When this image is compared to Fig. 2(a) (before near-field etching), significant decreases in flank roughness can be seen. To evaluate the flank roughness reduction, we digitized the AFM images in order to analyze them numerically. Figures 3(a) and (b) show the respective digitized AFM images of Figs. 2(a) and (b); the white and black areas correspond to land and groove areas, respectively. The images were rotated to align them with the $y$-axis along the corrugations of the nano-stripe pattern. We also evaluated the land widths of white areas in the digitized AFM images. Figures 3(c) and (d) show the respective land width $w$ distributions before and after near-field etching, which were least-square fitted by the black solid Gaussian curves. By comparing these fitted curves, we found that after near-field etching, the central value of the land width $w_{c}$ at the peak of the Gaussian curve decreased from $94.9 \mathrm{~nm}$ to $89.8 \mathrm{~nm}$, and the standard deviation $\sigma$ decreased from $20.7 \mathrm{~nm}$ to $17.6 \mathrm{~nm}$. These simultaneous decreases in $w_{c}$ and $\sigma$ indicate that near-field etching effectively reduced 
Fig. 2 Typical AFM images of the soda-lime glass (a) before and (b) after near-field etching (etching time $30 \mathrm{~min}$ )
Fig. 3 Digitized AFM images (a) before and (b) after near-field etching. Land width distributions (c) before and (d) after near-field etching

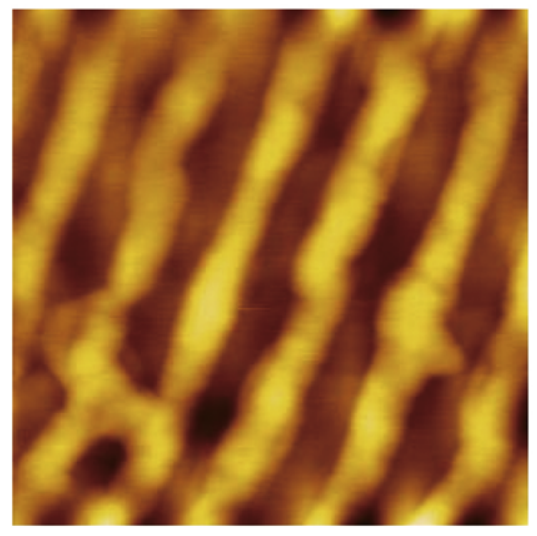

$500 \mathrm{~nm}$

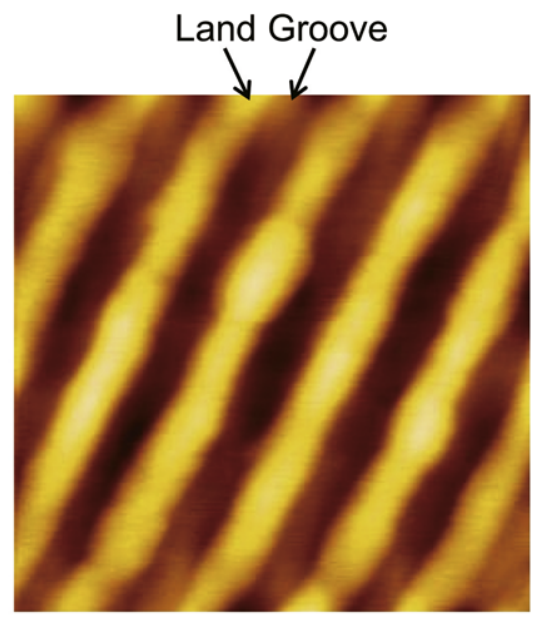

$500 \mathrm{~nm}$
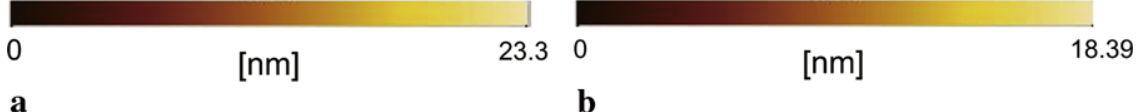


the flank roughness of the nano-stripe pattern. In addition to decreased flank roughness, a comparison of Figs. 3(c) and (d) confirms that land with width exceeding $125 \mathrm{~nm}$ disappeared, indicating that deburring is also realized by near-field etching. Furthermore, as Fig. 3(d) shows, the incidence of land with 90-nm width greatly exceeded the value of the fitted curve (the black solid curve), which also sug- gests that the deburring occurred (in other words, larger land was etched, and its width thereby decreased). Since the optical near-field was selectively generated at the protrusions, selective etching of larger land was accomplished by nearfield etching.

The roughness of the upper (land area) and lower (groove area) surfaces was also evaluated. We analyzed the surface 


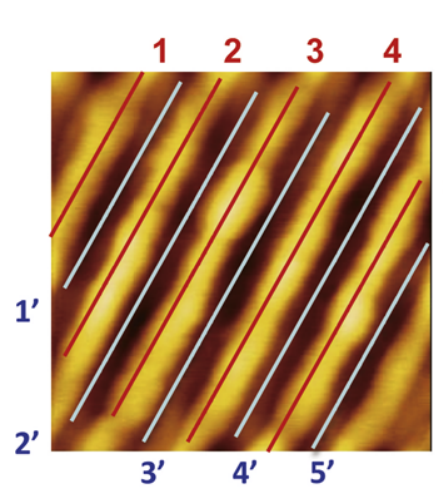

$\mathbf{a}$

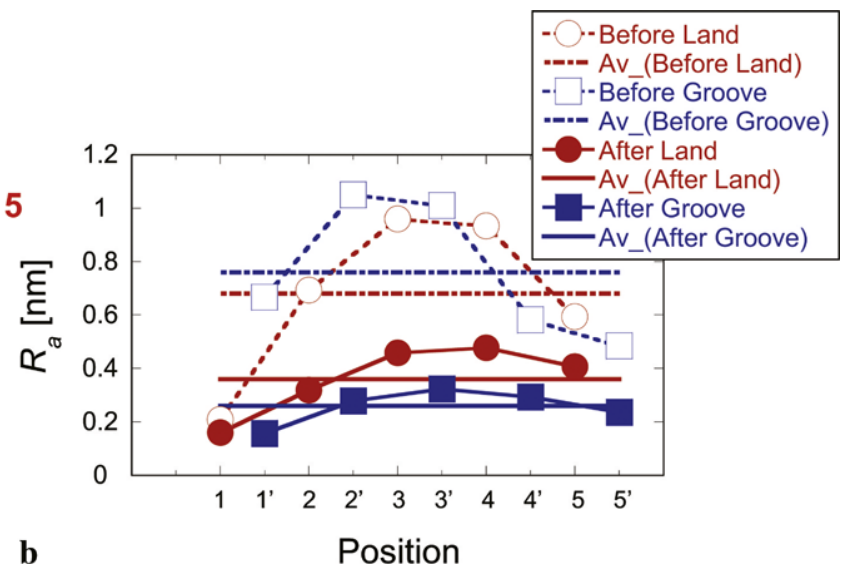

Fig. 4 (a) Schematic diagram of lines along land (solid lines) and grooves (dashed lines). (b) $R_{a}$ values before near-field etching along land (open circles and dashed line) and grooves (open squares and dashed line). $R_{a}$ values after near-field etching along land (solid circles and solid line) and grooves (solid squares and solid line). The number

roughness $R_{a}$ along land and grooves (Fig. 4(a)). Figure 4(b) summarizes the $R_{a}$ values before and after near-field etching. We found a significant reduction in $R_{a}$ along each line; the average $R_{a}$ for land decreased from $0.68 \mathrm{~nm}$ before etching (red dot-and-dashed line) to $0.36 \mathrm{~nm}$ after etching (red solid line), while the average $R_{a}$ for grooves decreased from $0.76 \mathrm{~nm}$ before etching (blue dot-and-dashed line) to 0.26 $\mathrm{nm}$ after etching (blue solid line). Additionally, from the averaged values of $R_{a}$, the etching rates for land and grooves were estimated to be 0.64 [nm/hour] and 1.0 [nm/hour], respectively, which were much larger than those of synthetic silica (0.14 [nm/hour]) [3]. Since soda-lime glass has a longer absorption band edge wavelength (350 nm) [10] than synthetic silica $(160 \mathrm{~nm})$ [11], this higher etching rate originated of near-field generation caused by the higher absorption coefficient of soda-lime glass.

\section{Conclusion}

We performed optical near-field etching of three-dimensional nano-stripe structures fabricated on soda-lime glass, with average height and period of $13.5 \mathrm{~nm}$ and $175 \mathrm{~nm}$, respectively. Thirty minutes of near-field etching reduced the flank roughness and surface roughness on both land and grooves. Since this technique is a non-contact method (i.e., without a polishing pad), it can be applied not only to the outer surfaces of three-dimensional substrates, but also to the inner wall surfaces of cylinders. Furthermore, the procedure is suitable for mass production.

of positions corresponds to the number of the lines shown in (a). Average $R_{a}$ : before etching of land—red dot-and-dashed line ( $\mathrm{Av} \_$(Before Land)); after etching of land_red solid line (Av_(After Land)); before etching of grooves - blue dot-and-dashed line (Av_(Before Groove)); after etching of grooves-blue solid line (Av_(After Groove))

Acknowledgements This work was partially supported by the New Energy and Industrial Technology Development Organization (NEDO) under the Research and Development Program of Innovative Energy Efficiency Technology and under the program of comprehensive activity for personnel training and industry-academia collaboration based on the NEDO project.

Open Access This article is distributed under the terms of the Creative Commons Attribution Noncommercial License which permits any noncommercial use, distribution, and reproduction in any medium, provided the original author(s) and source are credited.

\section{References}

1. B. Wua, A. Kumar, J. Vac. Sci. Technol. B 25, 1743 (2007)

2. L.M. Cook, J. Non-Cryst. Solids 120, 152 (1990)

3. T. Yatsui, K. Hirata, W. Nomura, M. Ohtsu, Y. Tabata, Appl. Phys. B 93, 55 (2008)

4. T. Yatsui, K. Hirata, Y. Tabata, W. Nomura, T. Kawazoe, M. Naruse, M. Ohtsu, Nanotechnology 21, 355303 (2010)

5. T. Kawazoe, K. Kobayashi, S. Takubo, M. Ohtsu, J. Chem. Phys. 122, 024715 (2005)

6. K. Kobayashi, T. Kawazoe, M. Ohtsu, IEEE Trans. Nanotechnol. 4, 517 (2005)

7. T. Yatsui, M. Ohtsu, Appl. Phys. Lett. 95, 043104 (2009)

8. R. Kullmer, D. Büerle, Appl. Phys. A 43, 227 (1987)

9. Y. Akita, Y. Kato, M. Hosaka, Y. Ono, S. Suzuki, A. Nakajima, M. Yoshimoto, Mater. Sci. Eng. B 161, 151 (2009)

10. L.B. Glebov, E.N. Boulos, J. Non-Cryst. Solids 242, 49 (1998)

11. L. Skuja, J. Non-Cryst. Solids 239, 16 (1998) 